\title{
Epidemiological approaches to 'epidemic neuromyasthenia': syndromes of unknown aetiology (epidemic myalgic encephalopathies)
}

\author{
MAIR ThOMAS \\ M.B., B.Sc., F.R.C.Path., M.F.C.M., D.P.H. \\ Epidemiological Research Laboratory, Central Public Health Laboratory, \\ Colindale Avenue, London NW9 5 HT
}

\begin{abstract}
Summary
The study of future outbreaks of 'epidemic neuromyasthenia' syndromes would be easier if there were a standing advisory arrangement for the co-ordination of basic standard studies, diagnostic tests and long term survey of patients. It is proposed that this might be achieved by establishing an 'EN Outbreaks Panel' at Colindale which, in addition to epidemiologists and virologists might include specialists in infectious diseases, neurology and psychiatry and other fields. The approach to such a panel would be through the Communicable Diseases Surveillance Centre (CDSC) when an outbreak is suspected. Arrangements could then be made by the CDSC with the Epidemiological Research Laboratory and the Virus Reference Laboratory to assist immediate investigations and for a follow-up to ascertain the incidence of sequelae, as well as for long storage of sera and other specimens.
\end{abstract}

\section{Introduction}

Both past and future outbreaks of epidemic neuromyasthenia syndromes have to be considered. Even when they seem alike the descriptions of past epidemics are seldom wholly comparable because differing sets of diagnostic measurement have been applied. A varied aetiology may be involved. Outbreaks have not been running a regular or predictable course like that of measles or whooping cough. Reports have been occasional, with some clustering at about 20-year intervals, perhaps because one account stimulates the publication of others. This may itself be related to the uncertainty of diagnosis.

\section{Clinical definition}

A precise clinical description of the disease concerned greatly assists epidemiological studies. The syndromes of 'epidemic neuromyasthenia' (EN) described have features suggestive of an organic disease of infective orgin, perhaps better described as epidemic myalgic encephalopathies. The nature of the outbreak suggests transmissibility with an incubation period from a few days to two or three weeks. The postulated agent is unknown.
Reports of epidemics indicate that the onset is often of non-specific malaise with little or no feverit There may also be transient sore throat, nausea or diarrhoea, and lymphadenopathy. This may be all and undiagnosable in the individual patient. However, associated patients may concurrently showo more characteristic features such as severe headache음 perhaps with vertigo, back pain, paraesthesiae,muscle tenderness and other muscle abnormalities $Z$ ranging from fasciculation to weakness of a limb or eye muscle and, on occasion, paresis. Such symptúms may be associated with marked psychological disturbance, in particular emotional lability, depression and lack of concentration. Recovery may take weeks, most patients have been well again withino $3{ }^{\circ}$ months, but relapses can occur after mild or severe. illness and may recur for years, particularly after fatigue, although some of these chronically affected persons ultimately improve and recover. Thus, unlikeڤ descriptions of multiple sclerosis and other chronic degenerative conditions of the nervous system, the $\vec{\rightarrow}$ course of even long standing cases of EN has tended 3 to be recurrent rather than progressive.

\section{Epidemiological studies}

Further systematic ascertainment of sequelae of 3 previously reported episodes might be rewarding, aso might the close study of sporadic cases, but a pro 3 . spective follow-up study of future outbreaks could be even more informative, and for this a system for the classification and study of this kind of illnesso would be useful. There is a tentative assumption $>$ that an undescribed aetiological agent is concerned, but at the same time it should be remembered that there are difficulties in relating a common infection? to an infrequent event, as the work on links between $N$ herpes virus and cancer of the cervix, or between $E^{N}$ virus and lymphoma have shown. If the investigationo of future outbreaks of EN-like syndromes can be co-ordinated, resemblances and differences between incidents should become apparent. One way in $\stackrel{?}{?}$ which this might be achieved would be by establish- 0 ing an 'Outbreaks Panel', possibly at Colindale. 
In any follow-up study it is essential to define the diagnostic criteria, and then to ascertain all new cases meeting the description within the population concerned. It may be enlightening to compare adjacent populations. In the first epidemic at the Royal Free Hospital in 1955, recognized cases were concentrated within three connected hospitals, but at the same time similar illness was occurring unquantified in the surrounding community. An outbreak is more likely to be diagnosed in the community if symptoms are severe, or relapses and sequelae frequent.

Evidently cases of epidemic disease are more readily labelled in institutions than elsewhere, in part because close and repeated contacts convey infection, and partly because the inconvenience of multiple illnesses within a short time span comes to administrative attention. In the community, however, epidemic infection may not be recognized unless a search is made. This may be because a condition is not distinctive like chickenpox or jaundice, but has a vague or wide clinical spectrum like $\mathrm{EB}$ virus or the EN syndrome; or because the morbidity rate is low, as among a population that is largely immune. Also, dispersion of cases may conceal an outbreak - the clientele of a restaurant which is distributing salmonellas may suffer at scattered addresses. By locating the homes, workplaces and recreational haunts of suspected cases it may be found that an epidemic which had seemed widespread is, in fact, centred on quite a limited number of foci, and may be curtailed by applying appropriate sanitary measures to a few such centres of infection.

The experience of Sonne dysentery surveillance by Thomas and Tillett (1973) in Enfield illustrates this. During an 18-year survey dysentery was never endemic in the school population and, even at times of epidemic incidence in the Borough, outbreaks were found to be localized within a very few primary schools, while secondary schools entirely escaped such outbreaks (Fig. 1).

There is a converse to this: if community standards of hygiene are low the apparent differential against institutions may be reversed and cholera or typhoid, for example, become epidemic in a village, but not in a residential school within that village.

\section{Lists and registers}

In hospitals, the clinical features of EN-like episodes are likely to be observed, investigated and recorded: hospital outbreaks might, therefore, be expected to provide good data for study, even retrospectively. Prospective studies in institutions or the community should have the advantage of consistent documentation and be supported by background registers of the population at risk and of cases as they arise. Such lists should include the age,

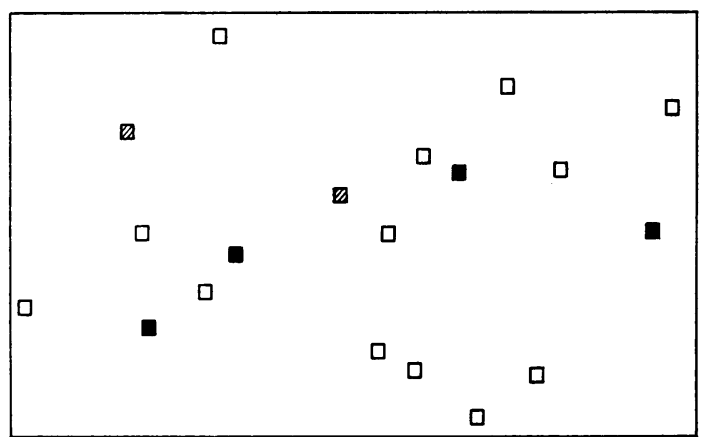

A

FIG. 1. Average incidence rates for Sonne dysentery according to the relative location of 19 primary schools: spring terms 1951-1968. Average number of cases per 100 pupils $\square 0-4 ; 05-9 ; \square 10+$. Scale: $A \rightarrow B=3 \cdot 2$ km.

sex, occupation, permanent address and sufficient other personal identification (such as National Insurance or passport number) from the outset to enable follow-up studies to be made many years later. These may be through, for example, home visits, professional directories, family practitioner committees, electoral rolls and death certification. Cases may become difficult to find through change of name or address and other causes unless there is a mechanism for keeping records up to date.

\section{Relation to follow-up studies to prognosis}

Prognosis is linked to the kind of patient referred for diagnosis as well as to the diagnostic criteria. Thus, the family doctor will tend to send his most ill patients into hospital, while, conversely, those least affected by the disease may not consult him. In general, the more ill the patient, the worse the prognosis, but this is not invariable with persistent infections. The captive population in an institutional outbreak is not analogous to that of patients sent into hospital by their general practitioner; it would be expected to include a higher proportion of cases with minimal symptoms, and might contain a number discovered by contact screening or laboratory tests and not in consequence of a complaint. For these reasons the results of following-up an institutional outbreak of a communicable disease can differ from those obtained by studying either hospital admissions or cases in the community, and the difference can be revealing.

The organization of the investigation of outbreaks of EN syndrome

This would fall naturally into two stages - an immediate field investigation and longer term studies. 
(1) The field investigation would follow the existing practice for an outbreak of communicable disease. The Medical Officer for Environmental Health, hospital Control of Infection Committee and institutional medical officers would be involved as appropriate in the community or institution concerned. The Communicable Diseases Surveillance Center (CDSC) at Colindale would like to be informed of suspected outbreaks at an early stage, either through the usual laboratory reporting system to the Communicable Disease Report (CDR) or independently, and would be glad to help at this stage or later.

It is proposed that the outbreak study co-ordinator would be an epidemiologist at the Epidemiological Research Laboratory.

Arrangements would be made to investigate patients using local diagnostic facilities initially, while ensuring that suitable laboratory specimens were stored for possible later study.

(2) Following this, if no known cause for the illness, such as an enterovirus, was discovered and the existence of an outbreak which appeared to be one of the EN syndrome was confirmed, then: arrangements would be made to set up a follow-ups study to ascertain the incidence of sequelae and foro the long-term storage of sera and other specimenso from a sufficient number of representative patients $\overline{\bar{\sigma}}$. (for example, a bank of well documented specimensक might be held at the Virus Reference Laboratorya for future examination).

To strengthen immediate and longer term studies $\overrightarrow{0}$ it might be useful to have an 'EN outbreaks advisory $\vec{\overrightarrow{ }}$ panel' of specialists, including a co-ordinator, an epidemiologist and a virologist, with an infectiouş diseases physician, a psychiatrist and a neurologist, 3 . and others whom such a panel might appoint.

\section{Reference}

Thomas, M.E M \& TILLETT, H.E (1973) Sonne dysentery in day schools and nurseries: an eighteen-year study inoo Edmonton. Journal of Hygiene. Cambridge, 71, 593. 